\title{
Cancellation of humming GSM mobile telephone noise
}

\author{
Ingvar Claesson \\ Blekinge Institute of Technology \\ SE 37225 Ronneby, Sweden
}

\author{
Andreas Nilsson \\ Ericsson Mobile Platform \\ SE 22100 Lund, Sweden
}

\begin{abstract}
A sometimes annoying problem in the most internationally widespread cellular telephone system, the GSM system, is an interfering signal generated by the switching nature of TDMA cellular telephone system. A humming noise originating from the speech frames, equivalent to 160 samples of data corresponding to $20 \mathrm{~ms}$ at $8 \mathrm{kHz}$ sampling rate is sometimes clearly audible.
\end{abstract}

This paper describes a study of two different software solutions designed to suppress such interference internally in the mobile handset. The methods are Notch Filtering, which is performed on a sample-per-sample basis, and Speech Frame Noise Cancellation, which is an alternative method employing correlators and subtraction, similar to Active Noise Control [2, 3].

\section{Introduction}

In GSM mobile telephony it is a common problem that an interfering signal is introduced into the microphone signal when the mobile is transmitting. This interfering signal is transmitted along with the speech signal to the receiver. Due to the humming sound of the interfering signal it is commonly denoted the Bumblebee.

Data from a speech frame of $20 \mathrm{~ms}$ is sent in several bursts, each occupying $1 / 8$ of a transmitting frame. The radio circuits are switched on and off with the radio access rate frequency. An electromagnetic field pulsating with this frequency and its harmonics disturbs its own microphone signal, as well as electronic equipment in the vicinity, producing in some cases annoying periodic humming noise in the uplink speech from the handset to the base station.

The interference consists of the fundamental frequency and its harmonics, where the fundamental switching rate is approximately $217 \mathrm{~Hz}$, more specifically, $5200 /(3 \cdot 8) \mathrm{Hz}$, according to the GSM standard [1]. Since the frequency components of the disturbing periodic humming noise are crystal generated and accurately known, it is possible to estimate the cosine- and the sine- parts of these with correlators also with high accuracy. This is easily done by correlating the microphone signal with sinusoids having the same crystal generated frequencies as the disturbing frequencies.This is an illustrative example of an application where subtraction of disturbances, typical for Active Noise Control $[2,3]$, is suitable.

\section{Signal Structure}

In GSM, Time Division Multiple Access (TDMA) is employed. The handheld mobile, formally denoted the mobile station (MS), sends information during a time slot that it is assigned and eight time slots constitutes a whole TDMAframe. The duration of a time slot is $3 / 5200$ seconds, and the period time of the TDMA-frames is $8 \cdot 3 / 5200$ seconds yielding the fundamental frequency is $1 /(8 \cdot(3 / 5200)) \approx 217$ $\mathrm{Hz}$ in Full Rate Transmission (FR).

In densely populated areas, such as Hong Kong, an alternative is sometimes used, Half Rate Transmission (HR), offering cheaper traffic with slightly decreased speech quality. In this case, the period of the interference is $1 /(8 \cdot 2$. $(3 / 5200)) \approx 108 \mathrm{~Hz}$, which is half the frequency of the FR, since the mobile is only transmitting during every other time slot, thus enabling almost twice the number of calls as compared to Full Rate Transmission.

A feature denoted Discontinuous Transmission (DTX) is also sometimes employed, allowing the radio transmitter to be switched off during speech pauses. During these pauses the background noise is averaged and only a Silent Descriptor (SID) is transmitted. SID frames hereby contain no disturbing frequencies, and consequently, the algorithm is not allowed to run during DTX.

\subsection{Analysis of the Bumblebee}

A typical recorded disturbed signal from a silent room can be seen in Figure 1. The interfering signal is periodic but somewhat complicated since, in the case of FR, there is no transmission when the mobile is listening to other base stations. Such silent frames occur once every 26 TDMAframes and are denoted idle frames, see Figure 2. In the HR case the disturbance pattern is even more complex, but observe that since the state of the communication between the mobile and base station is known, sufficient information to perform cancellation is always at hand.

A Fourier series expansion of the periodic disturbance 
Voltage[V]

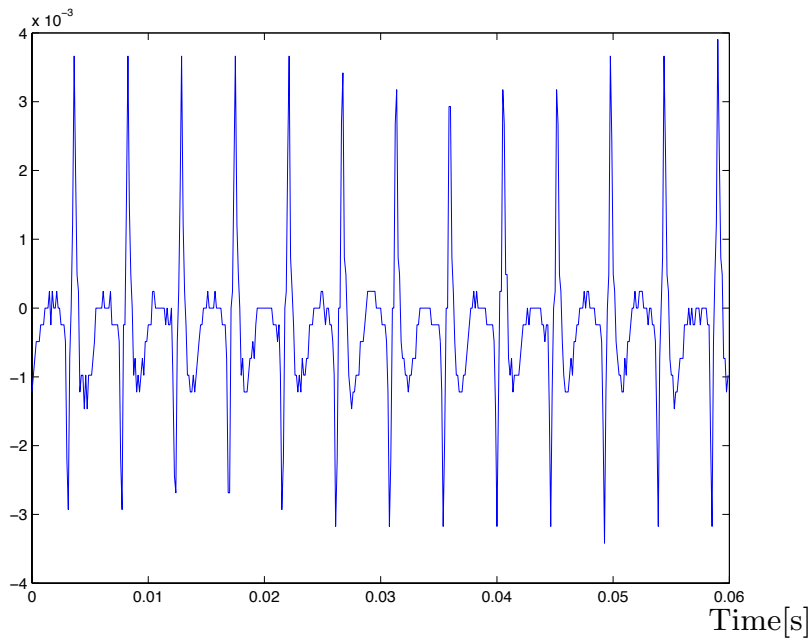

Figure 1: Interfering signal at the microphone $A / D$ converter recorded in a silent room with no speech.

yields

$$
x_{p}(n)=\sum_{k=1}^{K} C_{k} \sin \left(2 \pi k\left(f_{0} / f_{s}\right) n+\theta_{k}\right)
$$

where $K$ denotes the number of tones (fundamental plus harmonics), $f_{s}$ is the sample frequency, and $f_{0}$ represents the frequency of the fundamental tone. A similar Fourier series expansion can of course be carried out for the HR case. The number of tonal components $K$ that are needed depends of the bandwitdh, approximately $300-3400 \mathrm{~Hz}$, resulting in maximally $K=16$ tonal components in FR and $\mathrm{K}=32$ in $\mathrm{HR}$ transmission if the fundamental and upwards are to be covered.

\section{Solution proposals}

The methods to eliminate the Bumblebee disturbance are both working in the time domain. Linear Time-Invariant Notch filters, work on a sample-by-sample basis, while Noise Canceling Correlators, which work GSM frame-wise on 160 samples, corresponding to $20 \mathrm{~ms}$.

A notch filter contains deep notches, in its frequency response. Such a filter is useful when specific frequency components of known frequencies must be eliminated $[4,5]$. To eliminate the frequencies at $\omega_{n}, n=[1, \ldots, N]$, pairs of complex-conjugated nulls and zeros are placed on and just inside the unit circle at the angles $\omega_{n}$

$$
z_{n_{1,2}}=r_{b} e^{ \pm j \omega_{n}}, \quad r_{b}=1
$$

Consequently, the system function of the resulting notch filter is

$$
H(z)=\frac{B(z)}{A(z)}=b_{o} \prod_{n=1}^{N} \frac{\left(1-r_{b} e^{j \omega_{n}} z^{-1}\right)\left(1-r_{b} e^{-j \omega_{n}} z^{-1}\right)}{\left(1-r_{a} e^{j \omega_{n}} z^{-1}\right)\left(1-r_{a} e^{-j \omega_{n}} z^{-1}\right)}
$$

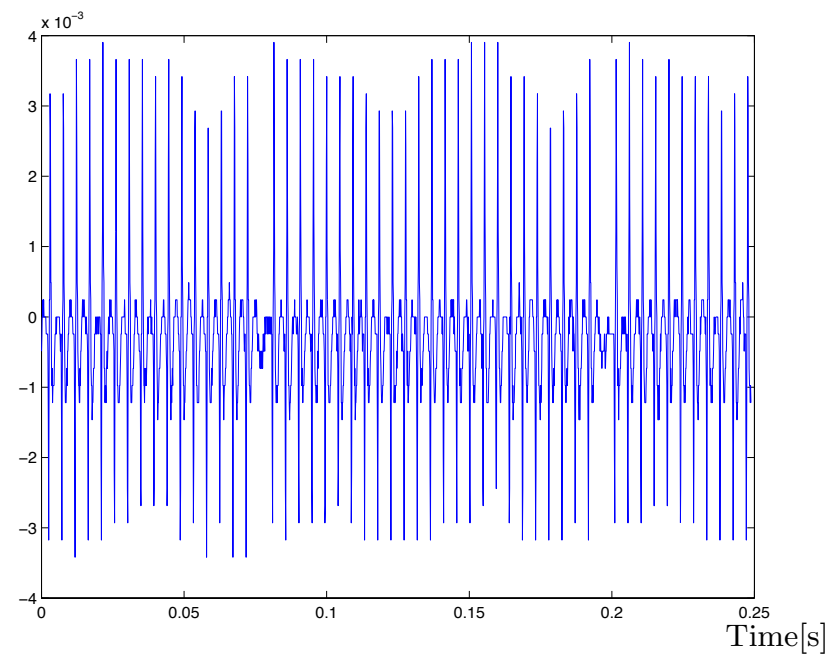

Figure 2: Pattern for interfering signal recorded in a silent room, Full Rate.

where

$$
b_{0}=\frac{\sum_{n=1}^{N} a_{n}}{\sum_{n=1}^{N} b_{n}}
$$

Alternatively, a band-limited periodic signal $x(k)$ can be represented by a finite sum of sinusoids.

$$
x(n)=\sum_{k=1}^{K} R_{k} \cos \left(2 \pi f_{k} n\right)+I_{k} \sin \left(2 \pi f_{k} n\right)+w(n)
$$

where $f_{k}=k \cdot f_{0}$ and $f_{0}$ is the fundamental frequency of the disturbance. Since the disturbance frequencies are known, only the coefficients of the cosine- and sine- parts, $R_{k}$ and $I_{k}$, need to be estimated.

The Maximum Likelihood (ML) estimate of known sinusoids in white noise background is given by correlation or matched filtering corresponding to finding the Fourier Expansion coefficients, or in the discrete-time case, the FFT coefficients at the exact frequencies where the periodic disturbances are. Even if speech cannot be regarded as a white disturbance, it is still an attractive Least Squares (LS) solution to correlate out the sinusoidals [6]-[8].

A sinusoidal correlator estimator consists mainly of a bank of dual product-adders, one for each frequency, one for each cosine- and sine part, in total $2 * K(K=16)$ correlators of length $\mathrm{N}=480$ in the full-rate case. This makes it easy to estimate and compensate the Bumblebee disturbance in "real time", frame by frame, by adding the correlation contribution of the most recent 160 samples, the present frame, and subtracting the correlation contribution of the 160 samples ( 3 frames back) in the frame leaving the estimation interval, i.e. the most recent 480 samples. The cosine- and sine- parts are estimated by correlation in accordance with Fig 3 , yielding the estimates $\hat{R}_{k}$ and $\hat{I}_{k}$, 
respectively in the two branches and are then subtracted from the input signal according to

$$
y(k)=x(k)-\sum_{k=1}^{K} \hat{R}_{k} \sin \left(2 \pi f_{k} n\right)+\hat{I}_{k} \sin \left(2 \pi f_{k} n\right)
$$

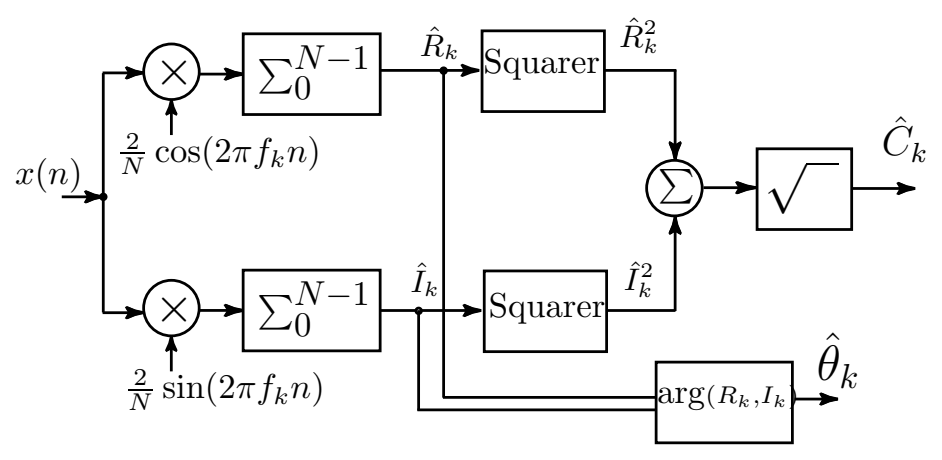

Figure 3: Sinusoidal estimation with correlators

The estimation should preferably be performed over an integer number of fundamental periods in order to avoid bias from incomplete periods. For the fundamental tone, which has the lowest frequency and thus requires most samples, we need 480 samples to fulfill the requirement in the FR case (and 960 samples in the HR case) since writing as a fractional number $f_{0} / f_{s}=13 / 480$ implies that 480 samples are needed to represent an integer number (13) of the fundamental periods with an integer number, i.e. 3 slots of 160 samples. We simply correlate the received signal with the $16^{*} 2$ basis functions of the correlators (cosines and sines) in order to obtain the coefficients for the cosines and sines. These estimates are subsequently used as coefficients for the amount each sinusoid should be subtracted from the received signal. No noise estimation is allowed during speech, since speech contains high energy at the same frequencies as the disturbance. Fortunately, a GSM mobile is already equipped with a VAD, which therefore can be easily utilized.

\subsection{Notch filter}

We first apply a notch filter directly in the signal path to reduce the interference. Implementation The notches are made as deep as possible, so that ideally the frequencies in question are totally eliminated. This results in the following system function:

$$
\frac{B(z)}{A(z)}=\frac{\sum_{k=1}^{16} a_{k}}{\sum_{k=1}^{16} b_{k}} \prod_{k=1}^{16} \frac{\left(1-r_{b} e^{j k \omega_{0}} z^{-1}\right)\left(1-r_{b} e^{-j k \omega_{0}} z^{-1}\right)}{\left(1-r_{a} e^{j k \omega_{0}} z^{-1}\right)\left(1-r_{a} e^{-j k \omega_{0}} z^{-1}\right)}
$$

The calculations are made recursively on the whole data set. This will result in a convergence period at the start up and also when a handover between base stations occurs. Unfortunately, the notch filter is active also under idle frames, a drawback resulting from the fact that it works sample-by-sample and recursively, leading to unwanted artifacts during idle frames, when trying to subtract a disturbance that is not present, i.e. a negative disturbance is added, see Figures 4-6.

It can be seen that the Bumblebee disturbance is considerably attenuated. However, this solution does not give a satisfactory result, since a portion of the speech is also attenuated, resulting in a "canned" or metallic sound. Another problem with this solution is that the periodic idle frame cannot be handled resulting in a new periodic interference, 26 times lower in frequency, see Figure 5. The reason for this is that the notch filter consists of poles (autoregressive), which give feedback of the output signal $(y(t))$ continuously. Consequently, the Bumblebee is added during the idle frame, according to the tails of impulse responses of IIR filters.

Power Spectrum $[\mathrm{dB}]$

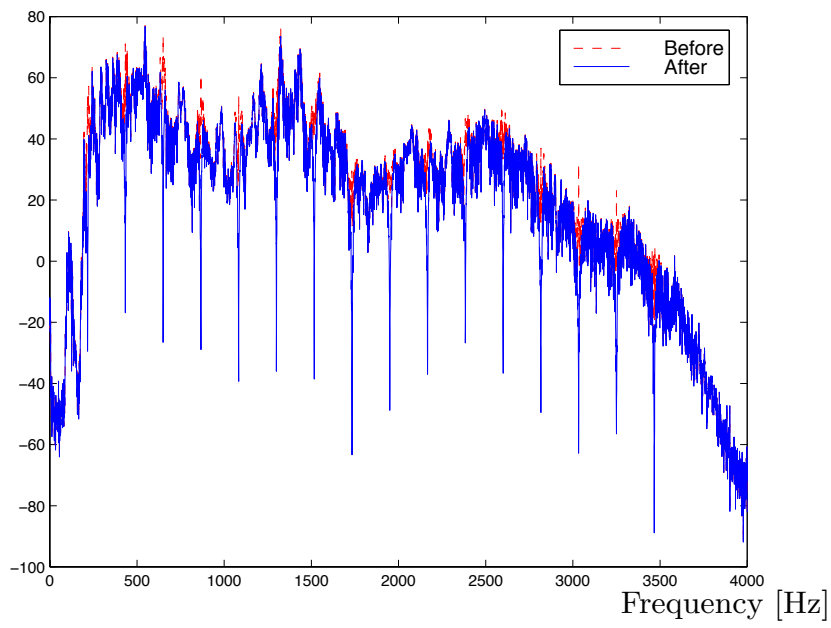

Figure 4: Cancellation of the Bumblebee with notch filter in speech [S1]. Full Rate, with speech.

\subsection{Correlators}

The data set that has been used is identical to that used when evaluating the notch filter, see Figure 7 . The metallic sound and the periodic interference that appeared in the notch tests from the idle frame are also avoided, thanks to time-limited subtractive nature of block correlation canceling, thus avoiding long-tailed (recursive) impulse responses. This gives a highly satisfactory result. Observe in Figures 7-8 that only the Bumblebee disturbance is attenuated. 
Voltage $[\mathrm{V}]$

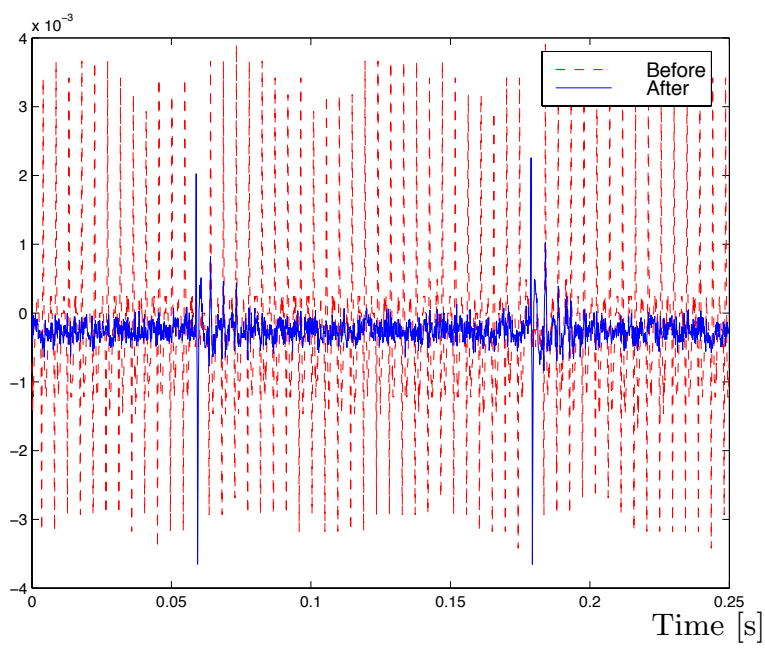

Figure 5: Time signal of the notched Bumblebee. Power Spectrum $[\mathrm{dB}]$

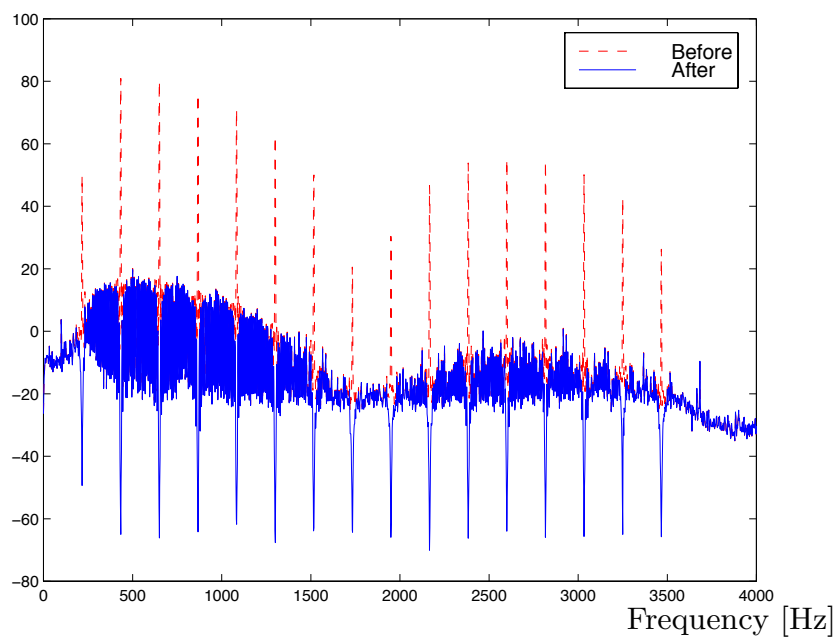

Figure 6: Cancellation of the Bumblebee with notch filter. The Bumblebee was recorded in a silent room [S2]. Full Rate, no speech.

\section{Complexity and Implementation aspects}

Complexity estimates have only been made for the correlators since this solution was preferred im MIPS, see Table 2 .

The complexity calculations are based on the attenuation of 16 sinusoids. The estimation is performed on 480 samples, and the subtraction of the estimated signal on 160 samples. This is the way it should be done in the mobile to avoid a delay. The sinusoids and the cosinusoids are stored in a Read Only Memory (ROM) as a table.

To form the 480 sine samples, a table should preferrably contain an integer number of periods for each frequency.
Power Spectrum $[\mathrm{dB}]$

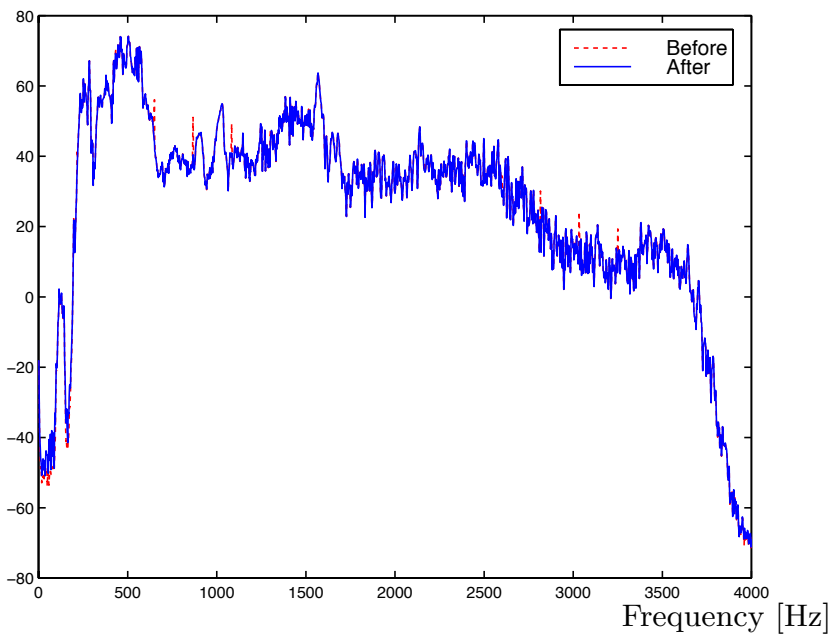

Figure 7: Cancellation of the Bumblebee in speech with correlators where VAD and idle mode have been taken into consideration [S3]. Full Rate, with speech.

That is, $480 / k$ samples with the exception of the frequencies stated in Table 1.

If $K=16$, a ROM of 6452 words, is required and the complexity is approximately 1.3 MIPS, see Table 2. Taking into account that control code and data transfers will also be needed, a very conservative estimation of the total complexity is 2 MIPS.We are certain that this figure can be considerably reduced. Symmetries in sinusoidal base functions and recursive estimation where the estimates are updated with the recent frame data of 160 samples can reduce the computational load by more than $50 \%$.

\begin{tabular}{|c|c|}
\hline $\mathbf{k}$ & Samples needed \\
\hline 7 & 480 \\
\hline 9 & 160 \\
\hline 11 & 480 \\
\hline 13 & 480 \\
\hline 14 & 240 \\
\hline
\end{tabular}

Table 1: Samples needed for the frequencies $k \cdot f_{0}$

\begin{tabular}{|l|l|l|}
\hline Task & Instructions / 20ms & MIPS \\
\hline Correlation & $16 \times 2 \times 480$ & 0.768 \\
Building $\hat{b}$ & $16 \times 2 \times 2 \times 160$ & 0.512 \\
Subtracting & 160 & 0.008 \\
\hline Total & $\mathbf{2 5 7 6 0}$ & $\mathbf{1 . 2 8 8}$ \\
\hline
\end{tabular}

Table 2: Complexity of the Table approach 
Power Spectrum $[\mathrm{dB}]$

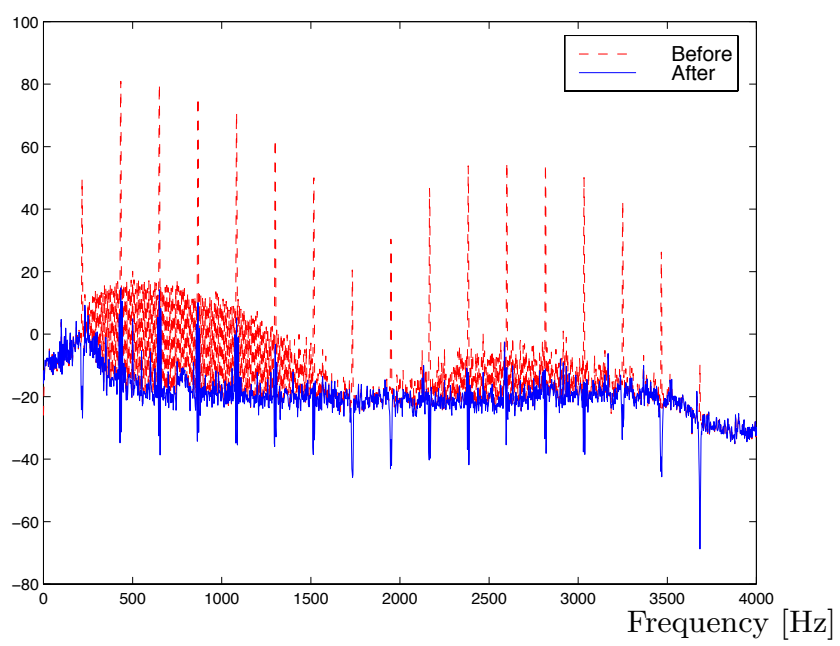

Figure 8: Cancellation of the Bumblebee with correlators where idle mode has been taken into consideration. The Bumblebee was recorded in a silent room [S4]. Full Rate, no speech.

\section{Summary, Conclusions and Fu- ture Work}

In this paper we have compared two methods for eliminating an annoying self-disturbance in mobile telephone microphone signals originating from the telephones's own antenna. Such disturbance is caused by TDMA switching in GSM cellular telephones. The GSM Frame-wise correlator approach which subtracts disturbances, instead of sampleby-sample filtering has shown great potential. The aim is now to implement the algorithm in fixed-point precision.

\section{References}

[1] GSM Standard (GSM 05.01 version 7.0.0 Release 1998) Digital cellular telecommunications system (Phase 2+), Physical layer on the radio path (General description).

[2] B.Widrow, S. D. Stearns Adaptive Signal Processing Prentice Hall, 1985

[3] M. Kuo, D. R. Morgan, Active Noise Control Systems, John Wiley \& Sons, Inc., 1996.

[4] Proakis, J.G. and Manolakis, D.G. Digital signal processing, pp. 343-345, 1996, Prentice-Hall Inc.

[5] Simon Haykin Digital communications, 1988, John Wiley \& Sons Inc.

[6] Peyton Z. Peebles, Jr. Probability, random variables, and random signal principles, 1993, McGraw-Hill Inc.
[7] Steven M. Kay Fundamentals of statistical signal processing: Estimation Theory, pp. 183-198, 1993, Prentice-Hall Inc.

[8] Per Eriksson On estimation of the amplitude and the phase function (Technical Report TR-148), 1981, University of Lund / SWEDEN.

A number of demo sound wav-files can be reached by searching the more exstensive Bumblebee killer report online at http://www.bth.se/fou/. Just search the archive with the keyword "bumblebee". All files are stored under mnemonic names.

[S1] Before and after Cancellation of the Bumblebee with Notch filter in speech.

[S2] Before and after Cancellation of the Bumblebee with Notch filter. No speech.

[S3] Before and after Cancellation of the Bumblebee with Correlators in speech where VAD and idle have been taken into consideration.

[S4] Before and after Cancellation of the Bumblebee with Correlators where the idle mode has been considered. No speech.

[S5] Before and after Cancellation of the Bumblebee with Correlators in the Half Rate case where the idle mode has been taken into consideration. No speech.

[S6] Before and after Cancellation of the Bumblebee with Correlators in speech in the Half Rate case where VAD and idle mode have been taken into consideration. 